\title{
Periodic solutions for a class of higher-order difference equations
}

\author{
Huantao Zhu ${ }^{1}$ and Weibing Wang ${ }^{2^{*}}$
}

\author{
* Correspondence: \\ gfwwbing@yahoo.com.cn \\ 2Department of Mathematics, \\ Hunan University of Science and \\ Technology, Xiangtan, Hunan \\ 411201, P.R. China \\ Full list of author information is \\ available at the end of the article
}

\section{Abstract}

In this article, we discuss the existence of periodic solutions for the higher-order difference equation

$$
x(n+k)=g(x(n))-f(n, x(n-\tau(n)) .
$$

We show the existence of periodic solutions by using Schauder's fixed point theorem, and illustrate three examples.

MSC 2010: 39A10; 39A 12.

Keywords: functional difference equation, periodic solution, fixed point theorem

\section{Introduction and main results}

Let $\mathbb{R}$ denote the set of the real numbers, $\mathbb{Z}$ the integers and $\mathbb{N}$ the positive integers. In this article, we investigate the existence of periodic solutions of the following highorder functional difference equation

$$
x(n+k)=g(x(n))-f(n, x(n-\tau(n)), \quad n \in \mathbb{Z},
$$

where $k \in \mathrm{N}, \tau: \mathbb{Z} \rightarrow \mathbb{Z}$ and $\tau(n+\omega)=\tau(n), f(n+\omega, u)=f(n, u)$ for any $(n, u) \in$ $\mathbb{Z} \times \mathbb{R}, \omega \in \mathbb{N}$.

Difference equations have attracted the interest of many researchers in the last 20 years since they provided a natural description of several discrete models, in which the periodic solution problem is always a important topic, and the reader can consult [1-7] and the references therein. There are many good results about existence of periodic solutions for first-order functional difference equations [8-12]. Only a few article have been published on the same problem for higher-order functional difference equations. Recently, using coincidence degree theory, Liu [13] studied the second-order nonlinear functional difference equation

$$
\Delta^{2} x(n-1)=f\left(n, x\left(n-\tau_{1}(n)\right), x\left(n-\tau_{2}(n)\right), \ldots, x\left(n-\tau_{m}(n)\right)\right),
$$

and obtain sufficient conditions for the existence of at least one periodic solution of equation (1.2). By using fixed point theorem in a cone, Wang and Chen [14] discussed the following higher-order functional difference equation

$$
x(n+m+k)-a x(n+m)-b x(n+k)+a b x(n)=f(n, x(n-\tau(n))),
$$

(c) 2011 Zhu and Wang; licensee Springer. This is an Open Access article distributed under the terms of the Creative Commons Attribution License (http://creativecommons.org/licenses/by/2.0), which permits unrestricted use, distribution, and reproduction in any medium, provided the original work is properly cited. 
where $a \neq 1, b \neq 1$ are positive constants, $\tau: \mathbb{Z} \rightarrow \mathbb{Z}$ and $\tau(n+\omega)=\tau(n), \omega, m, k \in \mathbb{N}$, and obtained existence theorem for single and multiple positive periodic solutions of (1.3).

Our aim of this article is to study the existence of periodic solutions for the higherorder difference equations (1.1) using the well-known Schauder's fixed point theorem. Our results extend the known results in the literature.

The main results of this article are following sufficient conditions which guarantee the existence of a periodic solution for (1.1).

Theorem 1.1. Assume that there exist constants $m<M, r>0$ such that $g \in C^{1}[m, M]$ with $r \leq g^{\prime}(u) \leq 1$ for any $u \in[m, M]$ and $f(n, u): \mathbb{Z} \times[m, M] \rightarrow \mathbb{R}$ is continuous in $u$,

$$
g(M)-M \leq f(n, u) \leq g(m)-m
$$

for any $(n, u) \in \mathbb{Z} \times[m, M]$, then (1.1) has at least one w-periodic solution $x$ with $m$ $\leq x \leq M$.

Theorem 1.2. Assume that there exist constants $m<M$ such that $g \in C^{1}[m, M]$ with $g^{\prime}(u) \geq 1$ for any $u \in[m, M]$ and $f(n, u): \mathbb{Z} \times[m, M] \rightarrow \mathbb{R}$ is continuous in $u$,

$$
g(m)-m \leq f(n, u) \leq g(M)-M
$$

for any $(n, u) \in \mathbb{Z} \times[m, M]$, then (1.1) has at least one w-periodic solution $x$ with $m$ $\leq x \leq M$.

\section{Some examples}

In this section, we present three examples to illustrate our conclusions.

Example 2.1. Consider the difference equation

$$
\begin{aligned}
& x(n+k)=a x(n)+q(n) \sqrt[3]{x(n-\tau(n))}, \\
& x(n+k)=b x(n)-q(n) \sqrt[3]{x(n-\tau(n))},
\end{aligned}
$$

where $k \in \mathbb{N}, 0<a<1, b>1, q$ is one $\omega$-periodic function with $q(n)>0$ for all $n \in$ $[1, \omega]$ and $\tau: \mathbb{Z} \rightarrow \mathbb{Z}$ and $\tau(n+\omega)=\tau(n)$.

Let $m>0$ be sufficiently small and $M>0$ sufficiently large. It is easy to check that

$$
\begin{aligned}
& (a-1) M \leq-q(n) \sqrt[3]{u} \leq(a-1) m \\
& (b-1) m \leq q(n) \sqrt[3]{u} \leq(b-1) M
\end{aligned}
$$

for $n \in \mathbb{Z}$ and $u \in[m . M]$. By Theorem 1.1 (Theorem 1.2), Equation (2.1) (or (2.2)) has at least one positive $\omega$-periodic solution $x$ with $m \leq x \leq M$. When $k=1$, this conclusion about (2.1) and (2.2) can been obtained from the results in [15]. Our result holds for all $k \in \mathbb{N}$.

Remark 1 Consider the difference equations

$$
\begin{aligned}
& x(n+k)=a x(n)+q(n) f(x(n-\tau(n))), \\
& x(n+k)=b x(n)-q(n) f(x(n-\tau(n))),
\end{aligned}
$$

where $k \in \mathbb{N}, 0<a<1, b>1, q$ is one $\omega$-periodic function with $q(n)>0$ for all $n \in$ $[1, \omega], \tau: \mathbb{Z} \rightarrow \mathbb{Z}$ and $\tau(n+\omega)=\tau(n)$ and $f:(0,+\infty) \rightarrow(0,+\infty)$ is continuous.

The following result generalizes the conclusion of Example 2.1. 
Proposition 2.1 Assume that $f_{0}=+\infty$ and $f_{\infty},=0$, here

$$
f_{0}=\lim _{u \rightarrow 0+} \frac{f(u)}{u}, \quad f_{\infty}=\lim _{u \rightarrow \infty} \frac{f(u)}{u},
$$

then (2.3) or (2.4) has at least one positive $\omega$-periodic solution.

Proof Here, we only consider (2.3). From $f_{0}=+\infty$ and $f_{\infty}=0$, we obtain that there exist $0<\rho_{1}<\rho_{2}$ such that

$$
f(u) \geq \frac{1-a}{\min q(n)} u, \quad 0<u \leq \rho_{1}, \quad f(u) \leq \frac{1-a}{\max q(n)} u, \quad u \geq \rho_{2} .
$$

Let $A=\min q(n) \min \left\{f(u): \rho_{1} \leq u \leq \rho_{2}\right\}$ and $B=\max q(n) \max \left\{f(u): \rho_{1} \leq u \leq \rho_{2}\right\}$. Choosing $\theta \in(0,1)$ such that

$$
\frac{A}{1-a} \geq \theta \rho_{1}, \quad \frac{B}{1-a} \leq \theta^{-1} \rho_{2}
$$

we obtain that

$$
\begin{gathered}
f(u) \geq \frac{1-a}{\min q(n)} u \geq \frac{\theta(1-a) \rho_{1}}{\min q(n)}, \quad \theta \rho_{1} \leq u \leq \rho_{1}, \\
f(u) \leq \frac{\theta^{-1}(1-a) \rho_{2}}{\max q(n)}, \quad \rho_{2} \leq u \leq \theta^{-1} \rho_{2}, \\
A \leq q(n) f(u) \leq B, \quad \forall n \in \mathbb{Z}, \rho_{1} \leq u \leq \rho_{2} .
\end{gathered}
$$

Using the above three inequalities, we have

$$
(1-a) \theta \rho_{1} \leq q(n) f(u) \leq(1-a) \theta^{-1} \rho_{2}, \quad \forall n \in \mathbb{Z}, \theta \rho_{1} \leq u \leq \theta^{-1} \rho_{2} .
$$

By Theorem 1.1, Equation (2.3) has at least one positive $\omega$-periodic solution $x$ with $\theta$ $\rho_{1} \leq x \leq \theta^{-1} \rho_{2}$.

Example 2.2. Consider the difference equation

$$
x(n+k)=-\frac{1}{x^{\alpha}(n)}+q(n)
$$

where $k \in \mathbb{N}, \alpha>0, q$ is one $\omega$-periodic function.

We claim that there is a $\lambda>0$ such that (2.5) has at least two positive $\omega$-periodic solutions for $\min q(n)>\lambda$.

In fact, $g(x)=-x^{-\alpha}$. Let $0<a<\sqrt[\alpha+1]{\alpha}$ be sufficiently small and $b>\sqrt[\alpha+1]{\alpha}$ be sufficiently large, then

$$
\begin{aligned}
& \frac{\alpha}{b^{\alpha+1}} \leq g^{\prime}(x)=\frac{\alpha}{x^{\alpha+1}} \leq 1, \quad \text { for } x \in[\sqrt[\alpha+1]{\alpha}, b] \\
& g^{\prime}(x)=\frac{\alpha}{x^{\alpha+1}} \geq 1, \quad \text { for } x \in[a, \sqrt[\alpha+1]{\alpha}] .
\end{aligned}
$$

If the following conditions are fulfilled

$$
\begin{aligned}
& -\frac{1}{b^{\alpha}}-b \leq-q(n) \leq-\frac{1}{\sqrt[\alpha+1]{\alpha^{\alpha}}}-\sqrt[\alpha+1]{\alpha}, \forall n \in \mathbb{Z}, \\
& -\frac{1}{a^{\alpha}}-a \leq-q(n) \leq-\frac{1}{\sqrt[\alpha+1]{\alpha^{\alpha}}}-\sqrt[\alpha+1]{\alpha}, \forall n \in \mathbb{Z},
\end{aligned}
$$


then (2.5) has at least one periodic solution $[a, \sqrt[\alpha+1]{\alpha}]$ and $[\sqrt[\alpha+1]{\alpha}, b]$ respectively. When $\min q(n)$ is sufficiently large, the conditions (2.6) and (2.7) are satisfied.

Example 2.3. Consider the difference equation

$$
x(n+k)=x^{3}(n)-2 x(n)-q(n) x^{2}(n-\tau(n)),
$$

where $k \in \mathbb{N}, q$ is one $\omega$-periodic function with $q(n)>0$ for all $n \in[1, \omega], \tau: \mathbb{Z} \rightarrow \mathbb{Z}$ and $\tau(n+\omega)=\tau(n)$.

Let $m=1, M>3+\max q(n)$ and $g(u)=u^{3}-2 u, f(n, u)=q(n) u^{2}$. It is easy to check that $g^{\prime}(u) \geq 1$ for $u \in[m, M]$, and

$$
g(m)-m=-2<f(n, u) \leq g(M)-M=M^{3}-3 M, \quad \forall n \in \mathbb{Z}, u \in[m, M] .
$$

By Theorem 1.2, Equation (2.8) has at least one positive $\omega$-periodic solution $x$ with $m \leq x \leq M$.

Remark 2 Consider the difference equation

$$
x(n+k)=g(x(n))-q(n) f(x(n-\tau(n))),
$$

where $k \in \mathbb{N}, q$ is one $\omega$-periodic function with $q(n)>0$ for all $n \in[1, \omega], \tau:{ }^{\prime} \rightarrow$ ' and $\tau(n+\omega)=\tau(n)$ and $f:(0,+\infty) \rightarrow(0,+\infty)$ is continuous.

Proposition 2.2 Assume that there exists $a>0$ such that $g \in C^{1}([a,+\infty), R)$ with $g^{\prime}(u) \geq 1$ for $u>a, f(u) \geq(g(a)-a) / \min q(n)$ for $u \geq a$. Further suppose that

$$
\lim _{u \rightarrow+\infty} \frac{g(u)-u}{f(u)}>\max q(n), \quad \lim _{u \rightarrow+\infty}(g(u)-u)=+\infty .
$$

Then (2.9) has at least one positive $\omega$-periodic solution.

Proof There exist $\rho>0$ such that

$$
g(u)-u \geq f(u) \max q(n), \quad u \geq \rho .
$$

Let $A=\min q(n) \min \{f(u): a \leq u \leq \rho\}$ and $B=\max q(n) \max \{f(u): a \leq u \leq \rho\}$. Since $\lim _{u \rightarrow+\infty}(g(u)-u)=+\infty$ and $g^{\prime}(u) \geq 1$ for $u>a$, there is $M>\rho$ such that $g(M)-M>B$ and

$$
f(u) \max q(n) \leq g(u)-u \leq g(M)-M, \quad \rho \leq u \leq M .
$$

Thus, (2.9) has at least one $\omega$-periodic solution $x$ with $a \leq x \leq M$.

\section{Proof}

Let $X$ be the set of all real $\omega$-periodic sequences. When endowed with the maximum norm $\|x\|=\max _{n \in[0, \omega-1]}|x(n)|, X$ is a Banach space.

Let $k \in \mathbb{N}$ and $0<c \neq 1$, and consider the equation

$$
x(n+k)=c x(n)+\gamma(n),
$$

where $\gamma \in X$. Set $(k, \omega)$ is the greatest common divisor of $k$ and $\omega, h=\omega /(k, \omega)$. We obtain that if $x \in X$ satisfies (3.1), then

$$
\begin{gathered}
c^{-1} x(n+k)-x(n)=c^{-1} \gamma(n), \\
c^{-2} x(n+2 k)-c^{-1} x(n+k)=c^{-2} \gamma(n+k), \\
\ldots \ldots \ldots \ldots \\
c^{-p} x(n+h k)-c^{1-p} x(n+(h-1) k)=c^{-p} \gamma(n+(h-1) k) .
\end{gathered}
$$


By summing the above equations and using periodicity of $x$, we obtain the following result.

Lemma 3.1. Assume that $0<c \neq 1$, then (3.1) has a unique periodic solution

$$
x(n)=\left(c^{-h}-1\right)^{-1} \sum_{i=1}^{h} c^{-i} \gamma(n+(i-1) k) .
$$

The following well-known Schauder's fixed point theorem is crucial in our arguments.

Lemma 3.2. [16] Let $X$ be a Banach space with $D \subset X$ closed and convex. Assume that $T: D \rightarrow D$ is a completely continuous map, then $T$ has a fixed point in $D$.

Now, we rewrite (1.1) as

$$
x(n+k)=p x(n)+[g(x(n))-f(n, x(n-\tau(n))-p x(n)],
$$

where $p>0$ is a constant which is determined later. By Lemma 3.1, if $x$ is a periodic solution of (1.1), $x$ satisfies

$$
x(n)=\left(p^{-h}-1\right)^{-1} \sum_{i=1}^{h} p^{-i}\left(H_{p} x\right)(n+(i-1) k),
$$

where $h=\omega /(k, \omega)$, the mapping $H_{p}$ is defined as

$$
\left(H_{p} x\right)(n)=g(x(n))-p x(n)-f(n, x(n-\tau(n)), x \in X .
$$

Define a mapping $T_{p}$ in $X$ by

$$
\left(T_{p} x\right)(n)=\left(p^{-h}-1\right)^{-1} \sum_{i=1}^{h} p^{-i}\left(H_{p} x\right)(n+(i-1) k), \quad x \in X .
$$

Clearly, the fixed point of $T_{p}$ in $X$ is a periodic solution of (1.1).

Proof of Theorem 1.1 Let $p=r$ and $\Omega=\{x \in X: m \leq x(n) \leq M$ for $n \in$ ', then $\Omega$ is a closed and convex set. If $r=1$, then $g(u)=u$ on $[m, M]$. It is easy to check that any constant $c \in[m, M]$ is a periodic solution of (1.1). Set $r<1$. Now we show that $T_{r}$ satisfies all conditions of Lemma 3.2. Noting that the function $g(u)-r u$ is nondecreasing in $[m, M]$, we have for any $x \in \Omega$,

$$
g(m)-r m \leq g(x(n))-r x(n) \leq g(M)-r M, \quad \forall n \in \mathbb{Z} .
$$

Let (1.4) be fulfilled. For any $x \in \Omega$ and $n \in \mathbb{Z}$,

$$
\begin{aligned}
\left(H_{r} x\right)(n) & =g(x(n))-p x(n)-f(n, x(n-\tau(n) \\
& \leq g(M)-r M-(g(M)-M) \\
& =(1-r) M, \\
\left(H_{r} x\right)(n) & =g(x(n))-p x(n)-f(n, x(n-\tau(n) \\
& \geq g(m)-r m-(g(m)-m) \\
& =(1-r) m .
\end{aligned}
$$


Hence, for any $x \in \Omega$ and $n \in \mathbb{Z}$,

$$
\begin{aligned}
\left(T_{r} x\right)(n) & =\left(r^{-h}-1\right)^{-1} \sum_{i=1}^{h} r^{-i}\left(H_{p} x\right)(n+(i-1) k) \\
& \leq\left(r^{-h}-1\right)^{-1} \sum_{i=1}^{h} r^{-i}(1-r) M=M, \\
\left(T_{r} x\right)(n) & =\left(r^{-h}-1\right)^{-1} \sum_{i=1}^{h} r^{-i}\left(H_{p} x\right)(n+(i-1) k) \\
& \geq\left(r^{-h}-1\right)^{-1} \sum_{i=1}^{h} r^{-i}(1-r) m=m .
\end{aligned}
$$

Hence, $T_{r}(\Omega) \subseteq \Omega$.

Since $X$ is finite-dimensional and $g(u), f(n, u)$ are continuous in $u$, one easily show that $T_{r}$ is completely continuous in $\Omega$. Therefore, $T_{r}$ has a fixed point $x \in \Omega$ by Lemma 3.2, which is a $\omega=$ periodic solution of (1.1). The proof is complete. $\square$

Proof of Theorem 1.2 Since $g \in C^{1}[m, M], \max \left\{g^{\prime}(u): m \leq u \leq M\right\}$ exists and max $\left\{g^{\prime}(u): m \leq u \leq M\right\} \geq 1$. Let $p=\max \left\{g^{\prime}(u): m \leq u \leq M\right\}$. If $p=1$, then $g(u) \equiv u$ on $[m$, $M]$. It is easy to check that any constant $c \in[m, M]$ is a periodic solution of (1.1). Next, we assume that $p>1$. Set $\Omega=\{x \in X: m \leq x(n) \leq M$ for $n \in \mathbb{Z}\}$. Noting that the function $g(u)-p u$ is nonincreasing in $[m, M]$, we have for any $x \in \Omega$,

$$
g(M)-p M \leq g(x(n))-p x(n) \leq g(m)-p m, \quad \forall n \in \mathbb{Z} .
$$

For any $x \in \Omega$ and $n \in \mathbb{Z}$,

$$
\begin{aligned}
\left(H_{p} x\right)(n) & =g(x(n))-p x(n)-f(n, x(n-\tau(n) \\
& \leq g(m)-p m-(g(m)-m) \\
& =(1-p) m, \\
\left(H_{p} x\right)(n) & =g(x(n))-p x(n)-f(n, x(n-\tau(n) \\
& \geq g(M)-p M-(g(M)-M) \\
& =(1-p) M .
\end{aligned}
$$

Hence, for any $x \in \Omega$ and $n \in \mathbb{Z}$,

$$
\begin{aligned}
\left(T_{p} x\right)(n) & =\left(p^{-h}-1\right)^{-1} \sum_{i=1}^{h} p^{-i}\left(H_{p} x\right)(n+(i-1) k) \\
& \geq\left(p^{-h}-1\right)^{-1} \sum_{i=1}^{h} p^{-i}(1-p) m=m, \\
\left(T_{p} x\right)(n) & =\left(p^{-h}-1\right)^{-1} \sum_{i=1}^{h} p^{-i}\left(H_{p} x\right)(n+(i-1) k) \\
& \leq\left(p^{-h}-1\right)^{-1} \sum_{i=1}^{h} p^{-i}(1-p) M=M .
\end{aligned}
$$

Hence, $T_{p}(\Omega) \subseteq \Omega$. $T_{p}$ has a fixed point $x \in \Omega$. The proof is complete. $\square$ 
Acknowledgements

The authors would like to thank the referee for the comments which help to improve the article. The study was supported by the NNSF of China (10871063) and Scientific Research Fund of Hunan Provincial Education Department (10B017).

\section{Author details}

${ }^{1}$ Hunan College of Information, Changsha, Hunan 410200, P.R. China ${ }^{2}$ Department of Mathematics, Hunan University of Science and Technology, Xiangtan, Hunan 411201, P.R. China

\section{Authors' contributions}

All authors contributed equally to the manuscript and read and approved the final manuscript.

\section{Competing interests}

The authors declare that they have no competing interests.

Received: 16 September 2011 Accepted: 23 December 2011 Published: 23 December 2011

\section{References}

1. Agarwal, RP: Difference Equations and Inequalities, 2nd edn.Marcel Dekker, New York (2000)

2. Antonyuk, PN, Stanyukovic, KP: Periodic solutions of the logistic difference equation. Rep Acad Sci USSR. 313, 1033-1036 (1990)

3. Berg, L: Inclusion theorems for non-linear difference equations with applications. J Differ Equ Appl. 10, 399-408 (2004). doi:10.1080/10236190310001625280

4. Cheng, S, Zhang, G: Positive periodic solutions of a discrete population model. Funct Differ Equ. 7, 223-230 (2000)

5. Zheng, B: Multiple periodic solutions to nonlinear discrete Hamiltonian systems. Adv Differ Equ (2007). doi: 10.1155/ 2007/41830

6. Zhu, B, Yu, J: Multiple positive solutions for resonant difference equations. Math Comput Model. 49, 1928-1936 (2009). doi:10.1016/j.mcm.2008.09.009

7. Zhang, X, Wang, D: Multiple periodic solutions for difference equations with double resonance at infinity. Adv Differ Equ (2011). doi:10.1155/2011/806458

8. Chen, S: A note on the existence of three positive periodic solutions of functional difference equation. Georg Math J. 18, 39-52 (2011)

9. Gil', Ml, Kang, S, Zhang, G: Positive periodic solutions of abstract difference equations. Appl Math E-Notes. 4, 54-58 (2004)

10. Jiang, D, Regan, DO, Agarwal, RP: Optimal existence theory for single and multiple positive periodic solutions to functional difference equations. Appl Math Comput. 161, 441-462 (2005). doi:10.1016/j.amc.2003.12.097

11. Padhi, S, Pati, S, Srivastava, S: Multiple positive periodic solutions for nonlinear first order functional difference equations. Int J Dyn Syst Differ Equ. 2, 98-114 (2009)

12. Raffoul, YN, Tisdell, CC: Positive periodic solutions of functional discrete systems and population model. Adv Differ Equ. 2005, 369-380 (2005)

13. Liu, Y: Periodic solutions of second order nonlinear functional difference equations. Archivum Math. 43, 67-74 (2007)

14. Wang, W, Chen, X: Positive periodic solutions for higher order functional difference equations. Appl Math Lett. 23 1468-1472 (2010). doi:10.1016/j.aml.2010.08.013

15. Raffoul, YN: Positive periodic solutions of nonlinear functional difference equations. Electron J Differ Equ. 2002, 1-8 (2002)

16. Guo, D, Lakshmikantham, V: Nonlinear Problem in Abstract Cones. Academic Press, New York (1988)

Cite this article as: Zhu and Wang: Periodic solutions for a class of higher-order difference equations. Advances in Difference Equations 2011 2011:66.

\section{Submit your manuscript to a SpringerOpen ${ }^{\circ}$ journal and benefit from:}

- Convenient online submission

Rigorous peer review

- Immediate publication on acceptance

- Open access: articles freely available online

- High visibility within the field

- Retaining the copyright to your article

Submit your next manuscript at $>$ springeropen.com 